\title{
Pathogen-associated acute encephalitis syndrome: therapeutics and management
}

\section{Future \\ MICROBIOLOGY}

\author{
Shailendra K Saxena ${ }^{* 1,2}$, Swatantra Kumar ${ }^{1} \&$ Vimal K Maurya ${ }^{1}$ \\ ${ }^{1}$ Center for Advanced Research (CFAR) - Stem Cell/Cell Culture Unit, Faculty of Medicine, King George's Medical University \\ (KGMU), Lucknow 226003, India \\ ${ }^{2}$ CSIR - Center for Cellular \& Molecular Biology, Uppal Road, Hyderabad 500007, India \\ *Author for correspondence: shailen@kgmcindia.edu
}

\begin{abstract}
"Effective treatment of pathogen-associated AES is based on the perfect diagnosis. Nevertheless, precise diagnosis is difficult to achieve due to the enormous range of etiological agents which lead to similar clinical manifestations."
\end{abstract}

First draft submitted: 1 December 2018; Accepted for publication: 17 January 2019; Published online: 13 February 2019

Keywords: acute encephalitis syndrome $\bullet$ complementary and alternative medicine $\bullet$ differential diagnosis $\bullet$ encephalitis $\bullet$ HSV encephalitis $\bullet$ Japanese encephalitis $\bullet$ vaccine

Worldwide recurrent cases of acute encephalitis syndrome (AES) have been attributed to various pathogens. Clinically, pathogen-associated AES is described as a severe onset of illness and an altered mental state with disorientation, an inability to focus, an inability to talk and some may present with an onset of seizures at any time, regardless of their age. Pathogen-associated AES can result from a multitude of pathogenic infections and patients who are diagnosed may present with a wide range of symptoms including severe illness, headache, reduced consciousness, vomiting, altered sensorium, tremors and convulsions [1]. Pathogen-associated AES can be differentiated from other etiologies of encephalopathy through considering its essential features: sudden onset of fever; cerebrospinal fluid comprising inflammatory cells or MRI-based confirmation and presence of pathogen or pathogen-specific antibodies.

Etiological agents of pathogen-associated AES can be broadly classified into six independent categories: viral, bacterial, parasitic, spirochetes, fungal, and their toxins. Japanese encephalitis virus (JEV) is the principal cause of viral AES in Southeast Asia [2]. Other predominant non-JEV viral agents are HSV, enteroviruses, coxsackievirus, dengue virus, Nipah virus (NiV) and HIV. Bacterial AES can be caused by Leptospira, Mycobacterium tuberculosis, Brucella, Orientia tsutsugamushi (Scrub typhus) and Ehrlichia chaffeensis [3]. Some parasites are also known to cause AES including Trypanosomes cruzi, Schistosoma mansoni, Toxoplasma gondii and Plasmodium falciparum [4].

\section{Diagnostic schemes for pathogen-associated AES}

The enormous diversity in etiological agents capable of causing AES, may result in intricate perfect diagnosis. In clinical practices, most cases are diagnosed based on clinical manifestation, lab reports, neuroimaging and electrophysiologic findings [5]. Suspected cases of pathogen-associated AES may be defined as a patient with neurological symptoms ranging from headache to meningitis or encephalitis with fever of variable severity. Symptoms may include fever, headache, stupor, meningeal signs, coma, disorientation, tremors, hypertonia, paralysis (generalized) and loss of coordination. Patients with fever or altered sensorium for more than $6 \mathrm{~h}$ with no skin rash may be included in the suspected cases of pathogen-associated AES. A probable case of pathogen-associated AES may be described as a suspected case with plausible laboratory results showing detection of pathogen-specific IgM antibody from serum taken during the acute phase of illness or higher and stable titers of pathogen-specific antibody determined by ELISA/HI/neutralizing assay. A confirmed case of pathogen-associated AES is through the detection of pathogen-specific IgM antibody in cerebrospinal fluid or indication of rise in paired sera from the acute and convalescent phases of illness through IgM/IgG, HI, ELISA, neutralization test or detection of pathogenic (virus,

Future $\because$ Medicine 
bacteria, parasite, spirochetes and fungi) genome or antigens in the blood or other body fluids including tissues through PCR, immunofluorescence or immunochemistry [6].

\section{Conventional treatments for pathogen-associated AES}

Effective treatment of pathogen-associated AES is based on the perfect diagnosis. Nevertheless, precise diagnosis is difficult to achieve due to the enormous range of etiological agents which lead to similar clinical manifestations. Some drugs have been shown to be effective against JEV, the well-documented causative agent of AES. However, none are currently available for patient use in a dose dependent manner. Minocycline, a tetracycline derivative, has been shown to be protective against JEV-induced AES, in one clinical trial [7]. The use of fluoxetine conferred protection against chronic enterovirus encephalitis [8]. Similarly, acyclovir has been shown to confer protection against HSV-induced encephalitis in patients, but due to resistance, other molecules are being tested [9]. Ribavirin has been shown to protect from NiV-induced acute encephalitis and recently favipiravir has been shown to be effective against $\mathrm{NiV}$ infection [10].

Unlike viral AES, treatment of bacterial AES has been established; however, the etiology should be confirmed at early stages of infection for optimal outcome. Neuro-leptospirosis can be differentiated primarily on asymptomatic basis. Along with aseptic meningitis, the other symptoms include myelopathy, myeloradiculopathy, meningoencephalitis, iridocyclitis and intracerebral bleed [11]. Crystalline penicillin is the drug of choice, whereas the role of steroids remains controversial. Similarly, tuberculous encephalopathy can be treated with a combination of antitubercular drugs and corticosteroids. Similarly, the use of antimicrobial agents such as doxycycline has been recommended for Orientia tsutsugamushi-induced encephalitis [12]. In cases of parasitic encephalitis, the use of benznidazole has been shown to be effective against Chagasic encephalitis caused by Trypanosomes cruzi [13]. The combinatorial treatment for encephalitis induced by Schistosoma mansoni infection comprised of praziquantel and artemether [14]. Unlike others, toxin-induced encephalitis is acute in nature and requires immediate treatment. At present, the overall management of pathogen-associated AES is based on symptomatic treatment and the scarcity of potential drugs suggests urgency for alternative preventive measures. The area of complementary and alternative (CAM) medicines provides great opportunity for developing potential treatments for encephalitis.

\section{Complementary \& alternative medicine for pathogen-associated AES}

Complementary and alternative medicine is progressively being used globally. Complementary medication is used alongside conventional medicines, while alternative strategies are used in place of mainstream medical health practices. CAM therapies may include a range of remedies coming from botanical, nutritional supplements and vitamins. CAM is a group of varied medical healthcare practices, systems and products, which are generally considered as 'nonmainstream' practices. The branch of integrative medicine combines both CAM and conventional treatments, based on evidence for safety and efficacy. In the USA, approximately $38 \%$ of adults and $12 \%$ of the children are using CAM in some form. CAM is already used extensively for medical conditions like musculoskeletal pain of neck, back and for joint pain. In order to manage AES, the use of CAM as an alternative approach might be advantageous.

Some plant products have been evaluated in vitro, for their efficacy and have been found to be potent candidate drugs for AES with potential for use as symptomatic therapeutics. Several antivirals such as bispidine, tilapia hepcidine, pentoxifylline, rosmarinic acid and luteolin have been shown as effective against JEV-induced AES, but none are available for patient use in a dose-dependent manner [15]. Astragalus polysaccharide from Astragalus membranaceus reduces the proliferation rate in HSV-1-induced astrocytes and augments the immunological functioning of the cells through the TLR3/NF-KB signaling pathway [16]. Cornus canadensis plant extract has been shown to exhibit antiviral activity against HSV-1, by acting as an attachment inhibitor [17]. Similarly, eupafolin from Kalanchoe gracilis has been used for its anti-enteroviral activity against coxsackievirus A16 and enterovirus 71. Additionally, it was found to be effective in reducing the virus-stimulated AP-1, STAT3 and ERK1/2 activation [18]. Furthermore, CAM has been extensively described against dengue infection and could be used for treating dengue-induced AES. Very recently, trials based on various ultra-diluted formulations, suggest the use of CAM for preventing AES. The drugs incorporated into the studies are belladonna, stramonium, arsenic album, sulfur, opium and nux vomica [19]. CAMs have been well described for mental disorders, which may be further considered for the management of AES. 


\section{Preventive strategies for pathogen-associated AES}

Preventive strategies of pathogen-associated AES depend on etiological complexity and the nature of the pathogen. SA-14-14-2, a live attenuated vaccine based on JEV is currently being used in China and nearby Asian countries, including India, Sri Lanka, Thailand and the Republic of Korea [20]. Nevertheless, the recurrent cases of Japanese encephalitis (JE) in the endemic areas suggest there is the need for a more effective vaccine. Some of the vaccines, such as IXIARO and ChimeriVax, are approved in some countries [21]. Additionally, a Vero cell-derived vaccine JENVAC has been developed using an Indian strain of JEV which has completed Phase III trial [22]. A vaccine candidate Kp r 56 for Orientia tsutsugamushi, has been developed that has lower efficiency for preventing re-infection but shows reduced levels of inflammation [23]. Several vaccines have been developed to prevent leptospirosis, such as recombinant protein vaccine, LPS vaccines, inactivated and attenuated vaccines and DNA based vaccines [24].

\section{Conclusions \& future perspectives}

Environmental, social and geographical factors such as global trade and travel, the expansive human population, altered ecosystems and microbial evolution all in tandem play an important role in disease transmission and outbreaks. Since etiological agents are broadly categorized, development of early diagnostics or prognostics capable of differentiating causative agents at the molecular level is imperative. Among all the etiological agents discussed, JEV is the most documented causative agent of AES. Broad spectrum antimicrobial agents targeting multiple pathogens are effective for developing ideal therapeutics for pathogen-associated AES. Prevention of pathogen-associated AES could be managed through the development of a peptide-based vaccine, based on consensus peptide sequences; development of such a multivalent vaccine is difficult to accomplish. Current vaccination programs are focusing on single pathogens such as Japanese encephalitis, with little success in significantly reducing the prevalence of disease, in endemic areas. CAM has been shown to be effective not only as therapeutics but also for preventive strategies.

The treatment of AES should rely on its etiology, which is indispensable for its overall management. Apart from vaccination, personnel protective measures are always considered as paramount for escaping infection. Several major objectives should be combined such as strengthening infectious disease surveillance and improving monitoring programs, network and surveillance systems to identify and respond to any infectious disease outbreaks. Public health infrastructure should be enhanced to support research and inquisition followed by implementation of effective control and preventive strategies.

\section{Financial \& competing interests disclosure}

The authors are grateful to the Vice Chancellor, King George's Medical University (KGMU), Lucknow and Director, Center for Cellular and Molecular Biology, Council of Scientific and Industrial Research (CSIR-CCMB), India for the encouragement for this work. SK Saxena is also supported by CCRH, Government of India, and US NIH grants: R37DA025576 and R01MH085259. The authors have no relevant affiliations or financial involvement with any organization or entity with a financial interest in or financial conflict with the subject matter or materials discussed in the manuscript. This includes employment, consultancies, honoraria, stock ownership or options, expert testimony, grants or patents received or pending, or royalties.

No writing assistance was utilized in the production of this manuscript.

\section{References}

Papers of special note have been highlighted as: $\bullet$ of interest

1 Ellul M, Solomon T. Acute encephalitis - diagnosis and management. Clin. Med. (Lond.) 18(2), 155-159 (2018).

2 Turtle L, Solomon T. Japanese encephalitis - the prospects for new treatments. Nat. Rev. Neurol. 14(5), 298-313 (2018).

- Discusses the worldwide recent advances in Japanese encephalitis and its management.

3 Glaser CA, Gilliam S, Schnurr D et al. In search of encephalitis etiologies: diagnostic challenges in the California Encephalitis Project, 1998-2000. Clin. Infect. Dis. 36(6), 731-742 (2003).

4 Granerod J, Ambrose HE, Davies NW et al. Causes of encephalitis and differences in their clinical presentations in England: a multicentre, population-based prospective study. Lancet Infect. Dis. 10(12), 835-844 (2010).

- Discusses the diverse etiological spectrum of encephalitis.

5 Venkatesan A, Geocadin RG. Diagnosis and management of acute encephalitis: a practical approach. Neurol. Clin. Pract. (3), 206-215 (2014).

- Suggests differential diagnosis and its importance for management of acute encephalitis syndrome.

6 Misra UK, Mani VE, Kalita J. A cost-effective approach to the diagnosis and management of acute infectious encephalitis. Eur. Neurol. 77(1-2), 66-74 (2017). 
7 Kumar R, Basu A, Sinha S et al. Role of oral minocycline in acute encephalitis syndrome in India - a randomized controlled trial. $B M C$ Infect. Dis. 16, 67 (2016).

8 Gofshteyn J, Cárdenas AM, Bearden D. Treatment of chronic enterovirus encephalitis with fluoxetine in a patient with X-linked agammaglobulinemia. Pediatr. Neurol. 64, 94-98 (2016).

9 Mitterreiter JG, Titulaer MJ, van Nierop GP et al. Prevalence of intrathecal acyclovir resistant virus in herpes simplex encephalitis patients. PLoS ONE 11(5), e0155531 (2016).

- Discusses about HSV-1 resistance to acyclovir suggesting that there is a need to look for alternative therapeutics.

10 Dawes BE, Kalveram B, Ikegami T et al. Favipiravir (T-705) protects against Nipah virus infection in the hamster model. Sci. Rep. 8(1), 7604 (2018).

11 Panicker JN, Mammachan R, Jayakumar RV. Primary neuroleptospirosis. Postgrad. Med. J. 77(911), 589-590 (2001).

- Discusses the clinical manifestation presented by neuroleptospirosis, which is very similar to encephalitis and should be included in the list etiology of acute encephalitis syndrome.

12 Murhekar MV. Acute encephalitis syndrome and scrub typhus in India. Emerg. Infect. Dis. 23(8), 1434 (2017).

13 Müller KJ, Garcia BF, Forsyth CJ et al. Clinical and pharmacological profile of benznidazole for treatment of Chagas disease. Expert Rev. Clin. Pharmacol. 19, 1-15 (2018).

14 Berkowitz AL, Raibagkar P, Pritt BS et al. Neurologic manifestations of the neglected tropical diseases. J. Neurol. Sci. 349(1-2), 20-32 (2015)

15 Saxena SK, Agrawal PT, Nair MPN. Current scenario of antiviral drugs for Japanese encephalitis. J. Med. Microb. Diagn. 3, 133 (2014).

- Discusses potential antivirals for Japanese encephalitis virus, targeting different stages of pathogenesis.

16 Shi L, Yin F, Xin X et al. Astragalus polysaccharide protects astrocytes from being infected by HSV-1 through TLR3/NF-B signaling pathway. Evid. Based Complement. Alternat. Med. 2014, 285356 (2014).

17 Lavoie S, Côté I, Pichette A et al. Chemical composition and anti-herpes simplex virus type 1 (HSV-1) activity of extracts from Cornus canadensis. BMC Complement. Altern. Med. 17(1), 123 (2017).

18 Wang CY, Huang SC, Lai ZR et al. Eupafolin and ethyl acetate fraction of Kalanchoe gracilis stem extract show potent antiviral activities against enterovirus 71 and coxsackievirus A16. Evid. Based Complement. Alternat. Med. 2013, 591354 (2013).

19 Oberai P, Varanasi R, Padmanabhan M et al. Effectiveness of homeopathic medicines as add-on to institutional management protocol for acute encephalitis syndrome in children: an open-label randomized placebo-controlled trial. Homeopathy 107(3), 161-171 (2018).

- The study suggests the use of several homeopathic medicines might be beneficial for acute encephalitis syndrome.

20 World Health Organization, Japanese encephalitis, Vaccine. www.who.int/ith/vaccines/japanese_encephalitis/en/

21 Li X, Ma SJ, Liu X et al. Immunogenicity and safety of currently available Japanese encephalitis vaccines: a systematic review. Hum. Vaccin. Immunother. 10(12), 3579-3593 (2014).

22 Singh A, Mitra M, Sampath G et al. A Japanese encephalitis vaccine from India induces durable and cross-protective immunity against temporally and spatially wide-ranging global field strains. J. Infect. Dis. 212(5), 715-725 (2015).

23 Chattopadhyay S, Jiang J, Chan TC et al. Scrub typhus vaccine candidate Kp r56 induces humoral and cellular immune responses in cynomolgus monkeys. Infect. Immun. 73(8), 5039-5047 (2005).

24 Wang Z, Jin L, Wegrzyn A. Leptospirosis vaccines. Microb. Cell Fact. 6, 39 (2007). 